\title{
Bosonic Dirty Paper Coding
}

\author{
Uzi Pereg \\ Institute for Communications Engineering, \\ Technical University of Munich, 80333 Munich, Germany. \\ Email: uzi.pereg@tum.de
}

\begin{abstract}
The bosonic channel is addressed with modulation interference and side information at the transmitter. The model can be viewed as the quantum counterpart of the classical random-parameter Gaussian channel. Based on Costa's writingon-dirty-paper result, the effect of the interference can be canceled. For both homodyne and heterodyne detection, we observe the same phenomenon, as the model reduces to a classical Gaussian channel. Then, we consider the bosonic channel with joint detection, for which the classical results do not apply, and derive a dirty-paper coding lower bound. We demonstrate that the optimal coefficient for dirty paper coding is not necessarily the MMSE estimator coefficient as in the classical setting.

Index Terms-Quantum communication, Shannon theory, channel capacity, dirty paper coding, state information.
\end{abstract}

\section{INTRODUCTION}

Optical communication forms the backbone of the Internet $[1,2,3]$. The bosonic channel is a simple quantummechanical model for optical communication over free space or optical fibers [4, 5]. An optical communication system consists of a modulated source of photons, the optical channel, and an optical detector. For a single-mode bosonic channel, the channel input is an electromagnetic field mode with an annihilation operator $\hat{a}$, and the output is another mode with the annihilation operator $\hat{b}$. The input-output relation in the Heisenberg picture is given by

$$
\hat{b}=\sqrt{\eta} \hat{a}+\sqrt{1-\eta} \hat{e}
$$

where $\hat{e}$ is associated with the environment noise and the parameter $\eta$ is the transmissivity, $0 \leq \eta \leq 1$, which depends on the length of the optical fiber and its absorption length [6] (see Figure 1). For a lossy bosonic channel, the noise mode $\hat{e}$ is in a Gibbs thermal state. Modulation is performed such that the unitary displacement operator $D(\alpha)=\exp \left(\alpha \hat{a}^{\dagger}-\alpha^{*} \hat{a}\right)$ is applied to the vacuum state $|0\rangle\langle 0|$. The bosonic channel can be viewed as the quantum counterpart of the classical channel with additive white Gaussian noise (AWGN) [4].

In classical communications, various settings of practical significance can be described by a channel $W_{Y \mid X}^{(s)}$ that depends on a random parameter $S \sim p_{S}$ when there is channel side information (CSI) available at the transmitter [7, 8]. For example, a cognitive radio in a wireless system may be aware of the channel state and network configuration [9]. Other applications include memory storage where the writer knows the fault locations [10], digital watermarking [11], and spreadspectrum communication [12], where the CSI represents the host data or a pseudo-random sequence to be modulated. The capacity of a random-parameter classical channel with CSI at the transmitter is given by [10]

$$
C(W)=\max _{p_{U, X \mid S}}[I(U ; Y)-I(U ; S)]
$$

where $U$ is an auxiliary random variable such that $U \odot(X, S) \bullet Y$ form a Markov chain.

A random-parameter Gaussian channel is specified by the input-output relation $Y=X+Z+S$, with a real-valued Gaussian noise $Z \sim \mathcal{N}_{\mathbb{R}}\left(0, \sigma_{Z}^{2}\right)$, an additive interference $S$ known to the transmitter, and an input power constraint $P$. A well-known result by Costa [13] is that the capacity of the random-parameter Gaussian channel is the same as if the interference is not there, i.e. $C(W)=\frac{1}{2} \log _{2}\left(1+\frac{P}{\sigma_{Z}^{2}}\right)$. Given that $S^{n}$ is not known to the receiver, it is far from obvious that the interference can be canceled out without sacrificing transmission power. The derivation of this result is based on Costa's dirty-paper coding (DPC) strategy [13]: Set

$$
U=X+t S
$$

such that $X$ is statistically independent of $S$. The optimal choice of the coefficient $t$ turns out to be the same as that of the minimum mean-square error (MMSE) estimator $\widehat{X}=t V$ for $X$ given an observation of $V=X+Z$ (see [7, Section 4.1]), namely,

$$
t=\frac{P}{P+\sigma_{Z}^{2}} .
$$

Explicit code constructions based on lattice codes were proposed in [14, 15] and references therein.

A quantum channel with random parameters is defined by a completely-positive trace-preserving map $\mathcal{E}_{A \rightarrow B}^{(s)}$ that depends on a classical random parameter $S \sim p_{S}$. At first, consider finite dimensions. Recently, the author [16] has determined that the capacity of the random-parameter quantum channel

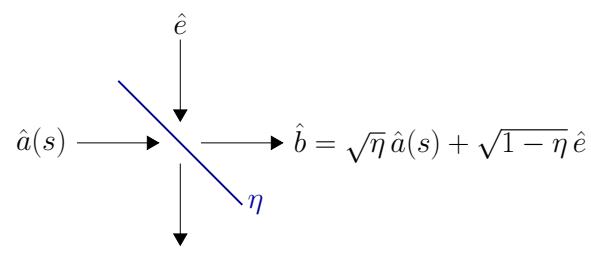

Fig. 1. The beam-splitter relation of the single-mode bosonic channel. 
with CSI at the transmitter is given by the regularized formula $C(\mathcal{E})=\lim _{n \rightarrow \infty} \frac{1}{n} \mathrm{C}_{1}\left(\mathcal{E}^{\otimes n}\right)$, with

$$
\mathrm{C}_{1}(\mathcal{E})=\sup _{p_{X \mid S}, \sigma_{A}^{x, s}}\left[I(X ; B)_{\rho}-I(X ; S)\right]
$$

where $I(X ; B)_{\rho}$ is the Holevo information calculated with respect to $\rho_{B}^{x, s}=\mathcal{E}_{A \rightarrow B}^{(s)}\left(\sigma_{A}^{x, s}\right)$. We extend this result to the bosonic channel with infinite-dimension Hilbert spaces based on the discretization limiting argument by Guha et al. [17]. Related settings were also considered in $[18,19,20]$.

In this work, we consider the single-mode lossy bosonic channel with a coherent-state protocol and a non-ideal displacement operation in the modulation process:

$$
\left|\zeta_{1} \zeta_{2} \cdots \zeta_{n}\right\rangle=D\left(\alpha_{1}+s_{1}\right)|0\rangle \otimes \cdots \otimes D\left(\alpha_{n}+s_{n}\right)|0\rangle
$$

where the parameter $s_{i}$ represents classical interference in the transmission equipment, which the transmitter becomes aware of, while the receiver is not. Alternatively, this can be viewed as a watermarking model with a quantum embedding. Given a classical host data sequence $s_{1}, \ldots, s_{n}$, Alice encodes an authentication message $m$ into a watermark $\left(\alpha_{i}\left(m, s_{1}, \ldots, s_{n}\right)\right)_{i=1}^{n}$. Next, Alice performs a quantum embedding of the watermark; she prepares a watermarked state $\left|\zeta_{1} \zeta_{2} \cdots \zeta_{n}\right\rangle$ as in (6), and transmits it to the authenticator Bob through the optical fiber. The capacity of the randomparameter bosonic channel represents the optimal rate at which the authenticator can recover the messages with high fidelity.

First, we consider homodyne and heterodyne detection. Both reduce to a classical random-parameter channel with either real or complex-valued Gaussian noise. Thereby, we observe that based on Costa's dirty-paper solution, the effect of the classical interference can be canceled, and the capacity is the same regardless of the intensity of the interference. Then, we consider joint detection, in which case, the problem does not reduce to that of a classical description. We derive a DPC lower bound based on the author's previous result [16], with a general coefficient $t$ (see (3)). Considering the special case of a pure-loss bosonic channel, we show that the optimal coefficient is not necessarily the MMSE value as in (4).

\section{Definitions}

We use the following notation; $X$ is a classical random variable, $p_{X}$ the probability density function; $\alpha$ is a complexvalued variable. $X \sim \mathcal{N}_{\mathbb{R}}\left(0, \sigma^{2}\right)$ indicates a real Gaussian variable, with $p_{X}(x)=\frac{1}{\sqrt{2 \pi \sigma^{2}}} e^{-x^{2} / 2 \sigma^{2}}$. A complex Gaussian $\alpha \sim \mathcal{N}_{\mathbb{C}}\left(0, \sigma^{2}\right)$ satisfies $\alpha=X+\mathrm{i} Y$ with independent $X, Y \sim \mathcal{N}_{\mathbb{R}}\left(0, \sigma^{2}\right)$. A quantum state of a system $A$ is denoted $\rho_{A}$. A channel $\mathcal{E}_{A \rightarrow B}$ is a cptp map that maps an input $\rho_{A}$ to an output $\rho_{B}$. A random-parameter quantum channel $\mathcal{E}_{A \rightarrow B}^{(s)}$ depends on a classical parameter $S \sim p_{S}$. The channel is memoryless, mapping $\rho_{A_{1} A_{2} \ldots A_{n}}$ to $\left(\bigotimes_{i=1}^{n} \mathcal{E}_{A_{i} \rightarrow B_{i}}^{\left(S_{i}\right)}\right)\left(\rho_{A_{1} A_{2} \ldots A_{n}}\right)$, where $S_{i}$ are i.i.d. $\sim p_{S}$. Given $\rho_{A B}$, define the von Neumann entropy $H(A)_{\rho}=-\operatorname{Tr}\left[\rho_{A} \log \left(\rho_{A}\right)\right]$, and let $I(A ; B)_{\rho}=$ $H(A)_{\rho}+H(B)_{\rho}-H(A B)_{\rho}$. A detailed description of bosonic systems can be found in [4]. The vacuum state of a singlemode electromagnetic field is denoted by $|0\rangle\langle 0|$. The creation operator $\hat{a}^{\dagger}$ creates an excitation: $\hat{a}^{\dagger}|0\rangle=|1\rangle, \hat{a}^{\dagger}|1\rangle=\sqrt{2}|2\rangle$, etc. Whereas, the annihilation operator $\hat{a}$ takes away an excitation. A coherent state $|\alpha\rangle$ corresponds to an oscillation of the bosonic field, obtained by $|\alpha\rangle=D(\alpha)|0\rangle$, where $D(\alpha) \equiv \exp \left(\alpha \hat{a}^{\dagger}-\alpha^{*} \hat{a}\right)$. A thermal state with a mean photon number $N$ is given by $\tau(N) \equiv \int_{\mathbb{C}} d^{2} \alpha \frac{e^{-|\alpha|^{2} / 2 N}}{\pi N}|\alpha\rangle\langle\alpha|$. A single-mode bosonic channel is described by the relation (1) between the input and output operators, $\hat{a}$ and $\hat{b}$, with an input constraint $N_{A}$. For a lossy channel, the noise mode $\hat{e}$ is in a thermal state $\tau\left(N_{E}\right)$, and for a pure-loss channel, in the vacuum state. Here, we consider the lossy bosonic channel with a coherent-state protocol and non-ideal modulation, as specified in (6). The coding definitions are given below.

Definition 1. An $(\mathrm{M}, n)$ code for the bosonic channel with a coherent-state protocol and CSI at the encoder consists of a set of $\mathrm{M}$ messages, an encoding map Enc : $\left(m, s_{1}, \ldots, s_{n}\right) \mapsto\left(\alpha_{i}\left(m, s_{1}, \ldots, s_{n}\right)\right)_{i=1}^{n}$, and a measurement Dec: $\rho_{B_{1}, \ldots, B_{n}} \mapsto \hat{M}$. The codewords satisfy $\left|\alpha_{i}\right|^{2} \leq$ $N_{A}$. Alice chooses a message $m$ from the message set. Using her access to the random parameters, she computes $\left(\alpha_{i}\left(m, s_{1}, \ldots, s_{n}\right)\right)_{i=1}^{n}$. The modulation produces the coherent state in (6), which is then sent through the channel. Bob receives the channel output systems $\left(B_{i}\right)_{i=1}^{n}$ and applies a measurement. The outcome $\hat{M}$ is his estimate of the message $m$. The coding rate is defined as $R=\frac{\log _{2}(\mathrm{M})}{n}$ in units of bits per transmission, and the maximal probability of error is denoted by $P_{e}^{(n)}=\max _{m} \operatorname{Pr}(\hat{M} \neq m \mid m)$. A rate $R>0$ is called achievable if there exists a sequence of $\left(\left\lceil 2^{n R}\right\rceil, n\right)$ codes such that $P_{e}^{(n)} \rightarrow 0$ as $n \rightarrow \infty$. The operational capacity $C(\mathcal{E})$ is defined as the supremum of achievable rates.

\section{RESUlTS}

First, we consider homodyne and heterodyne detection. A homodyne measurement of a quadrature observable is implemented in practice by combining the target quantum mode with an intense local oscillator at a 50:50 beam splitter, and measuring the photocurrent difference of the outgoing modes using two photodetectors [21]. When homodyne detection is used with a coherent-state protocol, the resulting channel $\mathcal{E}_{\text {hom }}$ is the random-parameter classical Gaussian channel

$$
Y=\sqrt{\eta}(\alpha+S)+Z_{\mathrm{hom}}
$$

with a real-valued Gaussian parameter $S \sim \mathcal{N}_{\mathbb{R}}\left(0, N_{S}\right)$ and noise $Z_{\text {hom }} \sim \mathcal{N}_{\mathbb{R}}\left(0, \frac{1}{4}\left[2(1-\eta) N_{E}+1\right]\right)$ [22]. Using the DPC scheme, we take $\alpha \sim \mathcal{N}_{\mathbb{R}}\left(0, N_{A}\right)$ and $U=\alpha+t_{1} S$ with $t_{1}=\frac{\eta N_{A}}{\eta N_{A}+\operatorname{var}\left(Z_{\text {hom }}\right)}$, such that $\alpha$ and $S$ are uncorrelated. The effect of the interference is thus removed, and the capacity is

$$
C\left(\mathcal{E}_{\text {hom }}\right)=\frac{1}{2} \log _{2}\left(1+\frac{4 \eta N_{A}}{2(1-\eta) N_{E}+1}\right)
$$

as without interference.

In heterodyne detection, two quadratures are measured by combining the measured mode with a vacuum mode into a 50:50 beam splitter, and homodyning the quadratures of the 


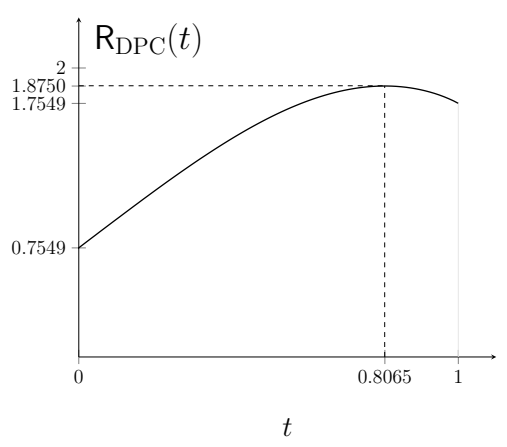

Fig. 2. The DPC lower bound for the pure-loss bosonic channel with joint detection and a coherent-state protocol, for an input constraint $N_{A}=2$, interference mean photon number $N_{S}=2$, and transmissivity $\eta=\frac{1}{2}$. The bound is based on the DPC assignment of the auxiliary random variables, $\alpha \sim \mathcal{N}_{\mathbb{C}}\left(0, \frac{1}{2} N_{A}\right)$ and $\gamma=\alpha+t S$. The figure depicts the lower bound $\mathrm{R}_{\mathrm{DPC}}(t)$ as a function of the DPC coefficient $t \in[0,1]$ (see (13)).

outcome modes [4]. Heterodyne detection is described by a random-parameter channel $\mathcal{E}_{\text {het }}$ with complex-valued Gaussian noise, specified by

$$
Y=\sqrt{\eta}(\alpha+S)+Z_{\text {het }}
$$

with a complex-valued circularly-symmetric Gaussian random parameter $S \sim \mathcal{N}_{\mathbb{C}}\left(0, \frac{1}{2} N_{S}\right)$ and noise $Z_{\text {het }} \sim \mathcal{N}_{\mathbb{C}}\left(0, \frac{1}{2}[(1-\right.$ 7) $\left.\left.N_{E}+1\right]\right)$ [22]. Similarly, we use DPC with $\alpha \sim \mathcal{N}_{\mathbb{C}}\left(0, \frac{1}{2} N_{A}\right)$ and $U=\alpha+t_{2} S$, with $t_{2}=\frac{\eta N_{A}}{\eta N_{A}+\operatorname{var}\left(Z_{\text {het }}\right)}$, achieving the capacity

$$
C\left(\mathcal{E}_{\text {het }}\right)=\log _{2}\left(1+\frac{\eta N_{A}}{(1-\eta) N_{E}+1}\right)
$$

as without interference.

For joint detection, the channel does not have a classical description. Applying the previous result by the author [16] for a quantum channel with random parameters, and using the DPC strategy, we obtain the lower bound $C\left(\mathcal{E}_{\text {joint }}\right) \geq \mathrm{R}_{\mathrm{DPC}}(t)$,

$$
\begin{aligned}
\mathrm{R}_{\mathrm{DPC}}(t) \equiv & I(\gamma ; B)-\left.I(\gamma ; S)\right|_{\gamma=\alpha+t S} \\
= & g\left(\eta\left(N_{A}+N_{S}\right)+(1-\eta) N_{E}\right) \\
& -g\left(\frac{\eta(1-t)^{2} N_{A} N_{S}}{N_{A}+t^{2} N_{S}}+(1-\eta) N_{E}\right) \\
& \quad-\log _{2}\left(\frac{N_{A}+t^{2} N_{S}}{N_{A}}\right)
\end{aligned}
$$

where $g(N)$ is the von Neumann entropy of the thermal state $\tau(N)$. That is, $g(N)=(N+1) \log _{2}(N+1)-N \log _{2}(N)$ for $N>0$, and $g(0)=0$. The second equality in (11) holds since $\zeta \equiv \alpha+S=\gamma+(1-t) S$, and the conditional variance of the channel parameter $S$ given $\gamma$ is

$$
\operatorname{var}(S \mid \gamma)=\left[1-\frac{(\operatorname{cov}(\gamma, S))^{2}}{\operatorname{var}(S) \operatorname{var}(\gamma)}\right] \operatorname{var}(S)=\frac{N_{A} N_{S}}{N_{A}+t^{2} N_{S}} .
$$

In particular, consider the special case of a pure-loss bosonic channel, where $N_{E}=0$. In this case,

$$
\begin{aligned}
\mathrm{R}_{\mathrm{DPC}}(t)= & g\left(\eta\left(N_{A}+N_{S}\right)\right)-g\left(\frac{\eta(1-t)^{2} N_{A} N_{S}}{N_{A}+t^{2} N_{S}}\right) \\
& -\log _{2}\left(\frac{N_{A}+t^{2} N_{S}}{N_{A}}\right) .
\end{aligned}
$$

To demonstrate, suppose that $N_{A}=N_{S}=2$ and $\eta=\frac{1}{2}$. Then, we have

$$
\mathrm{R}_{\mathrm{DPC}}(t)=g(2)-g\left(\frac{(1-t)^{2}}{1+t^{2}}\right)-\log _{2}\left(1+t^{2}\right) .
$$

Ignoring the CSI, we obtain a rate $\mathrm{R}_{\mathrm{DPC}}(t=0)=g(2)-$ $g(1)=3 \log _{2} 3-4=0.7549$. Whereas, using the DPC scheme with the MMSE coefficient $t_{0}=\frac{2}{2+0}=1$, we obtain a better rate: $\mathrm{R}_{\mathrm{DPC}}(t=1)=3 \log _{2} 3-2-\log _{2} 2=1.7549$. The optimal value for DPC turns out to be $t_{\max }=0.8065$, for which

$$
\mathrm{R}_{\mathrm{DPC}}\left(t_{\max }\right)=1.8750 \text {. }
$$

See Figure 2. The rate above is higher than the homodyne and heterodyne-detection capacities, $C\left(\mathcal{E}_{\text {hom }}\right)=1.1609$ and $C\left(\mathcal{E}_{\text {het }}\right)=1$, respectively. However, this rate is lower than the joint-detection capacity without interference $\left(N_{S}=0\right)$, which is given by $g(1)=2$.

Our results can be further extended to other optical channels. In particular, the random-parameter thermal amplifier channel $\mathcal{A}$ with an amplification gain $\kappa>1$ has the input-output relation $\hat{b}=\sqrt{\kappa} \hat{a}(s)+\sqrt{\kappa-1} \hat{e}^{\dagger}$ [23]. In a similar manner, we obtain the DPC lower bound

$$
\begin{array}{r}
C(\mathcal{A}) \geq \max _{t \in[0,1]}\left[g\left(\kappa\left(N_{A}+N_{S}\right)+(\kappa-1) N_{E}\right)\right. \\
-g\left(\frac{\kappa(1-t)^{2} N_{A} N_{S}}{N_{A}+t^{2} N_{S}}+(\kappa-1) N_{E}\right) \\
\left.-\log _{2}\left(\frac{N_{A}+t^{2} N_{S}}{N_{A}}\right)\right] .
\end{array}
$$

\section{DISCUSSION}

We conclude with the following remarks on the comparison between the classical and quantum settings:

1) Costa [13] provided the intuitive analogy of 'writing on dirty paper'. When a writer (Alice) is given a dirty paper, she knows the location and intensity of the dirt spots before writing. On the other hand, the reader (Bob) sees a mixture of the written text (channel input) and the dirt (channel parameter) without prior knowledge. In our setting, the dirt is the interference $s_{i}$ in the modulation displacement $D\left(\alpha_{i}+s_{i}\right)$. Alternatively, in the quantum watermarking scheme that we have described in the introduction, the dirt is the host covertext.

2) The classical capacity result can be derived using the DPC strategy in (3)-(4) following the observation that $U-t Y=X-t(X+Z)$ is the error of the MMSE estimation of $X$ given $V=X+Z$, hence it is statistically 
independent of the observation. Thereby, $(U-t Y)$ is jointly independent of $(V, S)$. This in turn implies that $(U-t Y)$ and $Y=V+S$ are statistically independent, leading to $H(U \mid Y)=H(U-t Y)=H(X \mid V)$ which can be used in order to show that

$$
I(U ; Y)-I(U ; S)=H(U \mid S)-H(U \mid Y)=I(X ; V)
$$

(see further details in [7] [24, Section 7.7]). For a bosonic channel with joint detection, we can also write the capacity in terms of $H(X \mid S)-H(X \mid B)_{\rho}$, with conditioning on the channel output. However, conditioning on a quantum system does not necessarily carry the meaning of an observation as in the classical setting [25].

3) While DPC was originally introduced to treat a channel with random parameters [13], the technique is useful in multi-user setups of wireless communications as well, such as the multiple-input multiple-output (MIMO) broadcast fading channel [26]. It is only natural to apply and extend our results to multi-mode bosonic networks.

\section{ACKNOWLEDGMENTS}

This work was supported by the German BMBF through grant n. 16KIS0856 and by the Israel CHE Fellowship for Quantum Science and Technology.

\section{REFERENCES}

[1] B. R. Bardhan and J. H. Shapiro. Ultimate capacity of a linear time-invariant bosonic channel. Phys. Rev. A, 93 (3):032342, 2016.

[2] I. Savov. Network information theory for classicalquantum channels. PhD thesis, McGill University, Montreal, 2012.

[3] S. Kumar and M. J. Deen. Fiber optic communications: fundamentals and applications. John Wiley \& Sons, 2014.

[4] C. Weedbrook, S. Pirandola, R. García-Patrón, N. J. Cerf, T. C. Ralph, J. H. Shapiro, and S. Lloyd. Gaussian quantum information. Rev. Mod. Phys., 84:621-669, May 2012.

[5] M. M. Wilde, P. Hayden, and S. Guha. Quantum tradeoff coding for bosonic communication. Phys. Rev. A, 86 (6):062306, 2012.

[6] J Eisert and MM Wolf. Gaussian quantum channels. In Quantum Inf. Cont. Variab. Atoms and Light, pages 2342. World Scientific, 2007.

[7] G. Keshet, Y. Steinberg, and N. Merhav. Channel coding in the presence of side information. Foundations and Trends in Communications and Information Theory, 4 (6):445-586, Jan 2007.

[8] U. Pereg and Y. Steinberg. The arbitrarily varying channel under constraints with side information at the encoder. IEEE Trans. Inf. Theory, 65(2):861-887, Feb 2019.

[9] A. Goldsmith, S. A. Jafar, I. Maric, and S. Srinivasa. Breaking spectrum gridlock with cognitive radios: An information theoretic perspective. Proc. of the IEEE, 97 (5):894-914, May 2009.
[10] C. Heegard and A. E. Gamal. On the capacity of computer memory with defects. IEEE Trans. Inf. Theory, 29(5):731-739, Sep 1983.

[11] P. Moulin and J. A. O'Sullivan. Information-theoretic analysis of information hiding. IEEE Trans. Inf. Theory, 49(3):563-593, Mar 2003.

[12] B. Chen and G. W. Wornell. Quantization index modulation: A class of provably good methods for digital watermarking and information embedding. IEEE Trans. Inf. Theory, 47(4):1423-1443, May 2001.

[13] M. Costa. Writing on dirty paper (corresp.). IEEE Trans. Inf. Theory, 29(3):439-441, May 1983.

[14] T. Philosof, U. Erez, and R. Zamir. Combined shaping and precoding for interference cancellation at low snr. In Proc. IEEE Int. Symp. Inf. Theory (ISIT'2003), page 68, 2003.

[15] U. Erez and S. ten Brink. A close-to-capacity dirty paper coding scheme. IEEE Trans. Inf. Theory, 51(10):34173432, 2005.

[16] U. Pereg. Communication over quantum channels with parameter estimation. In Proc. IEEE Int. Symp. Inf. Theory (ISIT'2020), pages 1818-1823, Jun 2020. URL https://arxiv.org/pdf/2001.00836.pdf.

[17] S. Guha, J. H. Shapiro, and B. I. Erkmen. Classical capacity of bosonic broadcast communication and a minimum output entropy conjecture. Phys. Rev. A, 76 (3):032303, 2007.

[18] H. Boche, N. Cai, and J. Nötzel. The classical-quantum channel with random state parameters known to the sender. J. Physics A: Math. and Theor., 49(19):195302, April 2016.

[19] A. Anshu, R. Jain, and N. A. Warsi. On the nearoptimality of one-shot classical communication over quantum channels. J. Math. Phys., 60(1):012204, 2019.

[20] U. Pereg, C. Deppe, and H. Boche. Quantum channel state masking. IEEE Trans. Inf. Theory, 67(4):22452268, 2021.

[21] S. L. Braunstein and P. van Loock. Quantum information with continuous variables. Rev. Mod. Phys., 77:513-577, Jun 2005.

[22] S. Guha. Multiple-user quantum information theory for optical communication channels. Technical report, MIT, 2008.

[23] V. Giovannetti, R. Garcia-Patron, N. J. Cerf, and A. S. Holevo. Ultimate classical communication rates of quantum optical channels. Nature Photonics, 8(10):796-800, 2014.

[24] A. El Gamal and Y.H. Kim. Network Information Theory. Cambridge University Press, 2011.

[25] M. Horodecki, J. Oppenheim, and A. Winter. Partial quantum information. Nature, 436(7051):673-676, 2005.

[26] H. Weingarten, Y. Steinberg, and S. S. Shamai. The capacity region of the gaussian multiple-input multipleoutput broadcast channel. IEEE Transactions on Information Theory, 52(9):3936-3964, 2006. 